\title{
An Approximate Solutions of two Dimension Linear Mixed Volterra- Fredholm Integral Equation of the Second Kind via Iterative Kernel Method
}

\section{Talaat Ismael Hasan}

Salahaddin University- College of Basic Education- Mathematic Department

E-mail: talhat.hassan@su.edu.krd

\begin{abstract}
:
In this work, we reformulate and apply iterative kernel method (IKM) for solving two dimension mixed Volterra-Fredholm integral equation of the second kind (MVFIE-2). The suitable algorithm for IKM is suggested and the programming for of the algorithm of the technique is written by Matlab programs. The computer application for the algorithm is tested on a number numerical examples. The results which are obtained by this technique compared with exact solution and some new theorems are proved; for decision the results computing the least square error (LSE) of the IKM and running time (RT) for the program.
\end{abstract}

Key words: Retia test, Iterative kernel method and MVFIE-2.

\section{Introduction:}

Integral equations have been one of the significant and principal instrument in various areas of sciences such as applied mathematical physics, biology and engineering. On the other hand, it has many applications in different areas of science, involving potential theory, electricity and quantum mechanics (Hasan, et al. 2016 \& Wazwaz, A.M. 2011).

In the last 15 years ago, many problems of integral equations was constructed from various cases in different events of the areas for applied sciences like mathematical physics, engineering and electromagnetic waves. Two dimension mixed Volterra- Fredholm integral equation of the second kind arises in many phenomena, physics and engineering areas (Mohammed K. S. 2015 \& Tenwich, M. C. 2012), as the of ordinary differential equations of two variables on the bounded region can be change and transform to module of two dimension mixed Volterra- Fredholm integral equation (Hafez et al. 2017 \& Zhong C. Z. and Jiang W. 2012).

Many scientists reformulated and applied different types of methods and techniques for getting the approximate solutions for these kinds of problems in integral equations, Jerri (1985) solved Volterra integral 
equations of the second kind by using IKM. Wazwaz (2007) used IKM for finding the approximate solution of Fredholm integral equation. Mahmud (2008) solved a system of linear two dimensional Fredholm integral equations of a second kind using Approximation iterative method. Zhong (2012) solved mixed linear VolterraFredholm integral equation. Berdawood (2015) solved mixed Volterra- Fredholm integral equations of the second type. Hasan (2016) used IKM for solving system of Volterra-Fredholm integral equations of the second kind. Saleh, et al. (2017) used fixed point method for obtaining the numerical solution for system VolterraFredholm integral equations of the second kind.

For extending this work, reformulating and using IKM for treating MVFIE-2 of two dimension for finding the approximate solution of it. Because, this method has a significant role for solving many problems in the area of integral equations.

Definition (1) The two dimension integral equation of the form

$$
u(x, t)=f(x, t)+\lambda \int_{a}^{t} \int_{\Omega} K(x, t, r, s) u(r, s) d r d s
$$

Is called mixed Volterra-Fredholm integral equation of the second kind, where $f(x, t)$ is given continuous functions on $E=\left\{(x, t) ; a \leq x \leq b, a \leq t \leq h_{1}\right\}$ and $K(x, t, r, s)$ is given continuous functions on the connected region $H=\{(x, t) ;(x, t) \in \Omega \times[0, T]\}$ while $u(x, t)$ is the unknown functions (Wazwaz, A.M. 2011).

Definition (2) (the Ratio test)

Let $\sum_{n=0}^{\infty} s_{n}(x)$ be a series with positive terms and suppose that $\operatorname{Lim}_{n \rightarrow \infty} \frac{s_{n+1}}{s_{n}}=L$ then

1- the series convergence if $L<1$.

2- the series divergence if $L>1$.

3- the series may converge or may diverge if $L=1$. (Berdawood, K. A. 2015 \& Mary C. 1979).

\section{The iterative kernel of Volterra integral equation of the second kind}

The Volterra integral equation of the second kind defined as:

$$
u(x)=f(x)+\int_{a}^{x} K(x, t) u(t) d t
$$

has iteration kernel method, let $K^{1}(s, t)=K(s, t)$

$$
K^{2}(x, y)=\int_{x}^{y} K(x, v) K^{1}(v, y) d v
$$

And $K^{3}(x, y)=\int_{x}^{y} K(x, v) K^{2}(v, y) d v$

in general the $\mathrm{n}^{\text {th }}$ iterative kernel for VIE-2 ${ }^{\text {nd }} \mathrm{k}$ ( Jerri, A. J. 1985 \& Wazwaz, A.M. 2011) is

$$
K^{n}(x, y)=\int_{x}^{y} K(x, v) K^{n-1}(v, y) d v, \text { for } n=1,2,3, \ldots m
$$




\section{Iterative kernel method for solving two dimension MVFIE-2}

The main idea of this work, is to reformulate iterative kernel method for solving MVFIE-2. Suppose that the approximate solution of equation (1) be as the form

$$
u(x, t)=\sum_{i=0}^{n} u^{i}(x, t)
$$

Substuting it in equation (1) and suppose that $K^{1}(x, t, r, s)=K(x, t, r, s)$ we get

$$
\sum_{i=0}^{n} u^{i}(x, t)=f(x, t)+\lambda \int_{a}^{t} \int_{\Omega} K(x, t, r, s) \sum_{i=0}^{n} u^{i}(r, s) d r d s
$$

By equaling the powers of $u^{i}(x, t)$, obtaining the following equations

$u^{0}(x, t)=f(x, t)$

and $u^{1}(x, t)$ formulate as

$u^{1}(x, t)=\lambda \int_{a}^{t} \int_{\Omega} K(x, t, r, s) u^{o}(r, s) d r d s=\lambda \int_{a}^{t} \int_{\Omega} K^{1}(x, t, r, s) f(r, s) d r d s$

$u^{2}(x, t)$ formulate as

$u^{2}(x, t)=\lambda \int_{a}^{t} \int_{\Omega} K(x, t, r, s) u^{1}(r, s) d r d s$

$\left.=\lambda \int_{a}^{t} \int_{\Omega} K(x, t, r, s) \lambda \int_{a}^{t} \int_{\Omega} K^{1}(v, w, r, s) f(r, s) d v d w\right] d r d s$

$=\lambda^{2} \int_{a}^{t} \int_{\Omega}\left[\int_{a}^{t} \int_{\Omega} K(x, t, v, w) K^{1}(v, w, r, s) d v d w\right] f(r, s) d r d s$

Such that $\left.K^{2}(x, t, r, s)=\int_{a}^{t} \int_{\Omega} K(x, t, v, w) K^{1}(v, w, r, s) d v d w\right]$

Then the second formula of iterative kernel is

$$
u^{2}(x, t)=\lambda^{2} \int_{a}^{t} \int_{\Omega} K^{2}(x, t, r, s) f(r, s) d r d s
$$

we find $u^{3}(x, t)$ by using the from, as

$$
\begin{aligned}
& \boldsymbol{u}^{3}(\boldsymbol{x}, \boldsymbol{t})=\lambda \int_{a}^{t} \int_{\Omega} K(\boldsymbol{x}, \boldsymbol{t}, \boldsymbol{r}, \boldsymbol{s}) \boldsymbol{u}^{2}(\boldsymbol{r}, \boldsymbol{s}) d \boldsymbol{r} d \boldsymbol{s} \\
& \left.=\lambda \int_{a}^{t} \int_{\Omega} K(x, t, r, s) \lambda^{2} \int_{a}^{t} \int_{\Omega} K^{2}(v, w, r, s) f(r, s) d v d w\right] d r d s
\end{aligned}
$$


$=\lambda^{3} \int_{a}^{t} \int_{\Omega}\left[\int_{a}^{t} \int_{\Omega} K(x, t, v, w) K^{2}(v, w, r, s) d v d w\right] f(r, s) d r d s$

Such that $\left.K^{3}(x, t, r, s)=\int_{a}^{t} \int_{\Omega} K(x, t, v, w) K^{2}(v, w, r, s) d v d w\right]$

Then $u^{3}(x, t)$ formulate as the form

$$
u^{3}(x, t)=\lambda^{3} \int_{a}^{t} \int_{\Omega} K^{3}(x, t, r, s) f(r, s) d r d s
$$

And the general formula of iterative method is

$$
u^{n}(x, t)=\lambda^{n} \int_{a}^{t} \int_{\Omega} K^{n}(x, t, r, s) f(r, s) d r d s
$$

Then general form of the $n^{\text {th }}$ iteration kernel is

$$
\left.K^{n}(x, t, r, s)=\int_{a}^{t} \int_{\Omega} K(x, t, v, w) K^{n-1}(v, w, r, s) d v d w\right]
$$

The numerical solutions which generate by this method $u(x, t)=\sum_{i=0}^{n} \boldsymbol{u}^{i}(x, t)$ approach to the exact solution of equation (1).

\section{Theorem (1)}

If $f(x, t)$ is bounded function on the connected rejoin $E=\left\{(x, t) ; a \leq x \leq b, a \leq t \leq h_{1}\right\}$ and $\boldsymbol{K}(\boldsymbol{x}, \boldsymbol{t}, \boldsymbol{r}, \boldsymbol{s})$ is bounded function on the rejoin $D=\left\{(x, t) ; a \leq r \leq x \leq b, a \leq s \leq t \leq h_{1}\right\}$, then the sequence $\left\{u^{n}(x, t)\right\}_{n=0}^{\infty}$ generated by the formula

$u^{n}(x, t)=\lambda^{n} \int_{a}^{t} \int_{a}^{b} K^{n}(x, t, r, s) f(r, s) d r d s$

is convergence uniformly to exact solution $\boldsymbol{u}(\boldsymbol{x}, \boldsymbol{t})$.

Proof: - By suppose $f(x, t)$ and $K(x, t, r, s)$ are bounded then there exist a positive numbers $e \geq \mathrm{O}$ and $d \geq \mathrm{O}$ such that $|f(x, t)| \leq e$ and $|K(x, t, r, s)| \leq d$ respectively. Now $u^{0}(x, t)=f(x, t)$, then $\left|u^{0}(x, t)\right|=|f(x, t)| \leq e$

Since $u^{1}(x, t)=\lambda \int_{a}^{t} \int_{\Omega} K^{1}(x, t, r, s) f(r, s) d r d s$

$$
\begin{aligned}
& \left|u^{1}(x, t)\right|=\left|\lambda \int_{a}^{t} \int_{a}^{b} K^{1}(x, t, r, s) f(r, s) d r d s\right|=|\lambda| \int_{a}^{t} \int_{a}^{b}\left|K^{1}(x, t, r, s)\right||f(r, s)| d r d s \\
& \left|u^{1}(x, t)\right| \leq|\lambda| \int_{a}^{t} \int_{a}^{b}(d)(e) d r d s=|\lambda| e d \int_{a}^{t} \int_{a}^{b} d r d s=|\lambda| e d(b-a) t
\end{aligned}
$$


Since we have $u^{2}(x, t)=\lambda^{2} \int_{a}^{t} \int_{\Omega} K^{2}(x, t, r, s) f(r, s) d r d s$,

then $\left|u^{2}(x, t)\right|=\left|\lambda \int_{a}^{t} \int_{a}^{b} K^{1}(x, t, r, s) u^{1}(r, s) d r d s\right|=|\lambda| \int_{a}^{t} \int_{a}^{b} K^{1}|(x, t, r, s)|(\lambda e d(b-a) s) d r d s$

$\left|u^{2}(x, t)\right|=\lambda \int_{a}^{t} \int_{a}^{b} d(\lambda e d(b-a) s) d r d s=\lambda^{2} e d^{2}(b-a) \int_{a}^{t} \int_{a}^{b} s d r d s$

$=\lambda^{2} e d^{2}(b-a)\left[\frac{(b-a) t^{2}}{2}\right]=\frac{\lambda^{2} e d^{2}(b-a)^{2} t^{2}}{2}$

$\left|u^{3}(x, t)\right|=\lambda \int_{a}^{t} \int_{a}^{b} d\left(\frac{\lambda^{2} e d^{2}(b-a)^{2} s^{2}}{2}\right) d r d s=\frac{\lambda^{3} e d^{3}(b-a)^{2}}{2} \int_{a}^{t} \int_{a}^{b} s^{2} d r d s$

$=\lambda^{3} e d^{3}(b-a)\left[\frac{(b-a) t^{3}}{3 !}\right]=\frac{\lambda^{2} e d^{2}(b-a)^{2} t^{3}}{3 !}$.

And so on, in general we obtain

$\left|u^{n}(x, t)\right|=\left|\lambda \int_{a}^{t} \int_{a}^{b} K(x, t, r, s) u^{n-1}(r, s) d r d s\right|=|\lambda| \int_{a}^{t} \int_{a}^{b} d\left(\frac{\lambda^{n-1} e d^{n-1}(b-a)^{n-1} t^{n-1}}{(n-1) !}\right) d r d s$

$\left|u^{n}(x, t)\right|=\frac{\lambda^{n} e d^{n}(b-a)^{n-1} t^{n-1}}{(n-1) !}[(b-a) t]=\frac{\lambda^{n} e d^{n}(b-a)^{n} t^{n}}{(n) !}$

By using ratio test, we want to show that the series

$|u(x, t)|=\sum_{i=0}^{n}\left|u^{i}(x, t)\right|=\sum_{i=0}^{\infty} \frac{\lambda^{i} e d^{i}(b-a)^{i} t^{i}}{(i) !}$ is convergence. Where the general term is

$R_{i}=\frac{\lambda^{i} e d^{i}(b-a)^{i} t^{i}}{(i) !}$

$\operatorname{Lim}_{i \rightarrow \infty} \frac{R_{i+1}}{R_{i}}=\operatorname{Lim}_{i \rightarrow \infty} \frac{\frac{\lambda^{i+1} e d^{i+1}(b-a)^{i+1} t^{i+1}}{(i+1) !}}{\frac{\lambda^{i} e d^{i}(b-a)^{i} t^{i}}{(i) !}}=\operatorname{Lim}_{i \rightarrow \infty} \frac{\lambda d(b-a) t}{(i+1)}=0$

Therefore, it is convergent for all values of $\lambda, d,(b-a)$ and $h_{1}$. the series is absolutely and uniformly convergent $\forall(x, t) \in D$.

Theorem (2) (Hafez et al. 2017)

Let $\boldsymbol{u}(\boldsymbol{x}, \boldsymbol{t})$ be a smooth function and $\boldsymbol{u}^{n}(\boldsymbol{x}, \boldsymbol{t})$ be the $\mathrm{n}^{\text {th }}$ approximate solution of $\boldsymbol{u}(\boldsymbol{x}, \boldsymbol{t})$ then $\left\|u(x, t)-u^{n}(x, t)\right\| \leq \frac{\lambda G}{(2 \pi)^{n}}$, where $\boldsymbol{G}$ is a positive constant independent of $\boldsymbol{n}$ and is a bound for the partial derivative of $f(x, t)$, such that $\pi=3.1428$.

\section{Theorem (3)}


Supposes that $\boldsymbol{u}(\boldsymbol{x}, \boldsymbol{t})$ be the exact solution of MVFIE-2, $\boldsymbol{f}(\boldsymbol{x}, \boldsymbol{t})$ is bounded function on $E=\left\{(x, t) ; a \leq x \leq b, a \leq t \leq h_{1}\right\}$ and $\boldsymbol{K}(\boldsymbol{x}, \boldsymbol{t}, \boldsymbol{r}, \boldsymbol{s})$ is bounded function on the rejoin $D=\left\{(x, t, s, t) ; a \leq r \leq x \leq b, a \leq s \leq t \leq h_{1}\right\}$ then a mapping $V: D \rightarrow E$ is contraction mapping.

Proof: - The $\mathrm{n}^{\text {th }}$ approximate solution has the form which is given in equation (8)

$$
u^{n}(x, t)=f(x, t)+\lambda \int_{a}^{t} \int_{a}^{b} K(x, t, r, s) u^{n-1}(r, s) d r d s
$$

Then we want to prove that $V$ is contraction mapping for sufficient large $n$,

$$
\begin{aligned}
& \left\|V(u(x, t))-V\left(u^{n}(x, t)\right)\right\|=\| f(x, t)+\lambda \int_{a}^{t} \int_{\Omega} K(x, t, r, s) u(r, s) d r d s-\left[f(x, t)+\lambda \int_{a}^{t} \int_{\Omega} K(x, t, r, s) u^{n-1}(r, s) d r d s \|\right. \\
& =\left\|\lambda \int_{a}^{t} \int_{\Omega} K(x, t, r, s)\left(u(r, s)-u^{n-1}(r, s)\right) d r d s\right\| \leq \lambda \int_{a}^{t} \int_{\Omega}\|K(x, t, r, s)\|\left\|u(r, s)-u^{n-1}(r, s)\right\| d r d s
\end{aligned}
$$

Since $\boldsymbol{K}(\boldsymbol{x}, \boldsymbol{t}, \boldsymbol{r}, \boldsymbol{s})$ is bounded function on $D$ then $|k(x, t, r, s)| \leq M$

$$
=\left\|\lambda \int_{a}^{t} \int_{\Omega} M\left[u(r, s)-u^{n-1}(r, s)\right] d r d s\right\| \leq \lambda M \int_{a}^{t} \int_{\Omega}\left\|u(r, s)-u^{n-1}(r, s)\right\| d r d s
$$

By using theorem (2), we have $\left\|u(x, t)-u^{n-1}(x, t)\right\| \leq \frac{\lambda G}{(2 \pi)^{n-1}}$

$$
\begin{aligned}
& \leq \lambda M \int_{a}^{t} \int_{\Omega} \frac{\lambda G}{(2 \pi)^{n-1}} d r d s \leq \lambda M \frac{\lambda G}{(2 \pi)^{n-1}} \int_{a}^{t} \int_{\Omega} d r d s \\
& \left\|V(u(x, t))-V\left(u^{n}(x, t)\right)\right\| \leq \frac{\lambda^{2} G M}{(2 \pi)^{n-1}} \int_{a}^{t} \int_{\Omega} d r d s
\end{aligned}
$$

Then for $n \rightarrow \infty$, we get $\frac{\lambda^{2} G M}{(2 \pi)^{n-1}} \int_{a}^{t} \int_{\Omega} d r d s \rightarrow 0$, Therefor $\left\|V(u(x, t))-V\left(u^{n}(x, t)\right)\right\| \rightarrow 0$

Hence, $V$ is contraction mapping.

\section{Algorithm of the technique IKM}

Input: $a, b, n, s, h_{1}, T o l$

Step 1: Suppose that the numerical solution of MVFIE-2 be $u(x, t)=\sum_{i=0}^{n} u^{i}(x, t)$

Step 2: Put $u^{0}(x, t)=f(x, t)$

For $i=0$ to $n$

Step 3: Using the relation in equation (9) for finding the iterative kernels.

Step 4: finding the component $u^{i}(x, t)$, by using equation (8).

Step 5: Computing the absolute error by using $e^{n}=\left|\sum_{i=0}^{n} u^{i}(x, t)-u(x, t)\right|$. 
If $e^{n}<$ Tol

Go to out put

End if

End for

Step 6: Containing in this process to get the approximate solution of MVFIE- $2^{\text {st }} \mathrm{K}$.

Output: the results of approximate solution and $e^{n}$.

\section{Numerical examples and results}

In this section, illustrating and discussing the IKM through numerical examples.

Example (1):- Find approximate solution of MVFIE-2 [2]

$$
u(x, t)=t^{2} e^{x}-\frac{2 x^{2} t^{3}}{3}+\int_{0}^{t} \int_{-1}^{1}\left(x^{2} e^{-y}\right) u(y, z) d y d z
$$

where the exact solutions $u(x, t)=t^{2} e^{x}$.

Solution. Applying the IKM on this problem, obtaining the following results.

Table 1: Comparison results between exact and approximate solutions.

\begin{tabular}{|c|c|c|c|c|}
\hline $\begin{array}{c}\text { The point } \\
(x, t)\end{array}$ & $\begin{array}{c}\text { Number of } \\
\text { iterations } \\
m\end{array}$ & $\begin{array}{c}\text { Exact solution } \\
\text { of } u(t, s)\end{array}$ & $\begin{array}{c}\text { Approximate } \\
\text { solutions } \\
\text { by IKM. }\end{array}$ & Absolute error \\
\hline$(0.4,0.3)$ & 1 & 0.21597740 & 0.33698866 & $1.2011 \times 10^{-1}$ \\
& 3 & & 0.26899877 & $5.3012 \times 10^{-2}$ \\
& 5 & & 0.21799786 & $2.0204 \times 10^{-3}$ \\
& 7 & & 0.21648988 & $5.1248 \times 10^{-4}$ \\
& 11 & 0.21595325 & $2.3752 \times 10^{-5}$ \\
& 13 & & 0.21595806 & $1.8934 \times 10^{-6}$ \\
& 15 & & 0.21597748 & $4.8432 \times 10^{-7}$ \\
& 17 & & 0.21597745 & $5.3581 \times 10^{-8}$ \\
& 21 & & 0.21597740 & $4.1238 \times 10^{-9}$ \\
& & & 0.21597740 & $2.4672 \times 10^{-11}$ \\
& & & & \\
\hline
\end{tabular}

Table 2: Show LSE and RT according to the number of iterations

\begin{tabular}{|c|c|c|}
\hline $\begin{array}{c}\text { Number of } \\
\text { iterations }\end{array}$ & LSE & RT \\
\hline 3 & $3.6503 \times 10^{-4}$ & $0: 0: 1.5375$ \\
7 & $4.7639 \times 10^{-8}$ & $0: 0: 3.6823$ \\
11 & $5.4471 \times 10^{-12}$ & $0: 0: 6.2131$ \\
15 & $5.1248 \times 10^{-15}$ & $0: 0: 8.7601$ \\
\hline
\end{tabular}




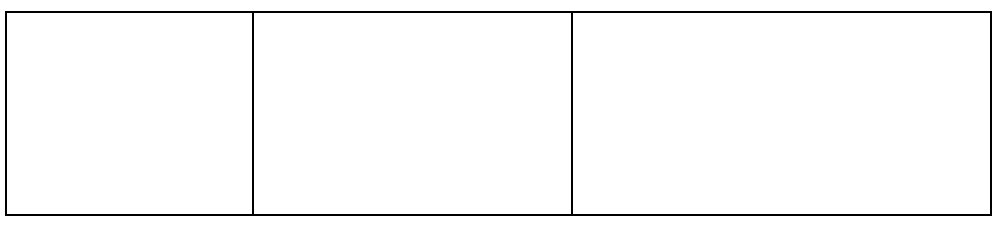

Example (2):- Find approximate solution of MVFIE-2 [12]

$$
u(x, t)=e^{-t}\left[\cos (x)+t \cos (x)+\frac{1}{2} t \cos (x-1) \sin (1)\right]-\int_{0}^{t} \int_{0}^{1}\left(\cos (x-y) e^{z-t}\right) u(y, z) d y d z
$$

where the exact solutions $u(x, t)=e^{-t} \cos (x)$.

Solution. Applying the IKM on this problem, obtaining the following results.

Table 3: Comparison results between exact and approximate solutions.

\begin{tabular}{|c|c|c|c|c|}
\hline $\begin{array}{c}\text { The } \\
\text { point } \\
(x, t)\end{array}$ & $\begin{array}{c}\text { Number of } \\
\text { iterations } m\end{array}$ & $\begin{array}{c}\text { Exact solution } \\
\text { of } u(t, s)\end{array}$ & $\begin{array}{c}\text { Approximat } \\
\text { e solutions } \\
\text { by IKM. }\end{array}$ & Absolute error \\
\hline$(0.4,0.3)$ & 1 & 0.64038119 & 1.72546123 & 1.08508001 \\
& 3 & & 0.68854761 & $3.1551 \times 10^{-1}$ \\
& 5 & & 0.63568923 & $4.8162 \times 10^{-2}$ \\
& 7 & & 0.64507309 & $4.6919 \times 10^{-3}$ \\
& 11 & & 0.64095741 & $5.7622 \times 10^{-4}$ \\
& 13 & & 0.64041212 & $3.0912 \times 10^{-5}$ \\
& 15 & & 0.64038652 & $5.3343 \times 10^{-6}$ \\
& 19 & & 0.64038165 & $4.6324 \times 10^{-7}$ \\
& 21 & & 0.64038143 & $2.4211 \times 10^{-8}$ \\
& 19 & & 0.64038119 & $4.9657 \times 10^{-10}$ \\
& $15.2124 \times 10^{-12}$ \\
\hline
\end{tabular}

Table 4: Show LSE and RT according to the number of iterations.

\begin{tabular}{|c|c|c|}
\hline $\begin{array}{c}\text { Number of } \\
\text { iterations }\end{array}$ & LSE & RT \\
\hline 3 & $4.2312 \times 10^{-3}$ & $0: 0: 1.4260$ \\
7 & $5.3012 \times 10^{-7}$ & $0: 0: 2.9743$ \\
11 & $2.0204 \times 10^{-11}$ & $0: 0: 5.8762$ \\
15 & $5.1248 \times 10^{-14}$ & $0: 0: 8.0302$ \\
\hline
\end{tabular}

\section{Conclusions :}

In this work, iterative kernel method was reformulated for finding the approximate solutions of two dimension MVFIE-2 discussed. This method was applied for getting the numerical results on the numerical examples for this type of problems, this technique gives very good results for this kind of the problems and we 
proved the convergence of the method. From tables (1) and (3) shown a comparison between the exact and numerical solutions. In addition, in tables (2) and (4) computing the LSE and RT which are criterion of discussion. Finally, from theorems (1), (3) and the results which showed in tables, we conclude that the effecting of this technique and it was successively for solving this problems.

\section{Bibliography:}

Abdou M. A. and Abdul Kader G . M. (2005) Mixed type of Fredholm Volterra integral equation. Journal LE MATEMATICHE. Vol. LX (I), 41-58.

Berdawood, K. A. (2015). Computational method for solving mixed Volterra Fredholm integral equation of the second kind, MSc thesis Salahaddin University.

Ibrahim, H. Attah, F. and Gyegwe, T. (2016). On the solution of Volterra-Fredholm and mixed VolterraFredholm integral equations using the new iterative method. Journal of applied Mathematics. 6(1), 1-5.

Hafez R. M., Doha E. H., Bhrawy A. H. and Baleanu D. (2017). Numerical solutions of two dimension mixed Volterra- Fredholm integral equation of the second kind via Bernoulli collocation method. Romanian Journal of Physics, 62(111), 1-11.

Hasan, T. I. Sulaiman N. A. and Saleh S. (2016). Aitken method on iterative kernel method for solving system of Volterra-Fredholm integral equations of the second kind. Journal of Pure and Applied Sciences (ZJPAS), 28(6), 79-88.

Jerri, A. J. (1985). Introduction to integral equations with applications. Marcel Dekker. New York and Basel.

Mahmud, M.H. (2008). Approximation methods for solving system of linear two dimensional Fredholm integral equations of a second kind. Master thesis Salahaddin University.

Mary C. (1979). Calculus and Analytic Geometry. Fifth edition, Addison Wesley publishing.

Mohammed K. S. (2015). Numerical Solution for Mixed Volterra-Fredholm Integral Equations of The Second Kind By Using Bernstein Polynomials Method. Journal Mathematical Theory and Modeling. 5 (10), 154-162.

Saleh S., Hasan, T. I. and Sulaiman N. A. (2017). Fixed point and its improvement for the system of Volterra-Fredholm integral equations of the second kind. Journal of Mathematica. 33(2), 191-206.

Tenwich, M. C. (2012). Error estimates for numerical solutions of one and two dimension Integral equations, PhD Thesis, University of Leeds department of applied Mathematics.

Wazwaz, A.M. (2011). Linear and non-linear integral equations methods and applications. Springer-Verlag Berlin Heidelberg.

Zhong C. Z. and Jiang W. (2012). An approximate solution for a mixed linear Volterra-Fredholm integral Equation. Journal Applied Mathematics and letter, 25(1), 1131-1134. 
في هذا البحث قمنا بصياغة و تطبيق الطريقة التقريبة التكرارية لحل لمعادلة فولتير ا- فريدهولم التكاملية التركيبية

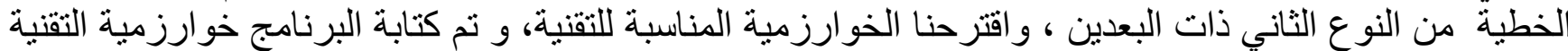

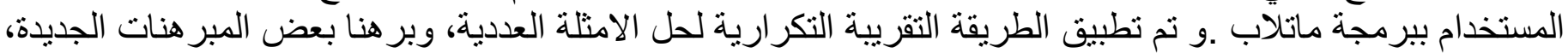

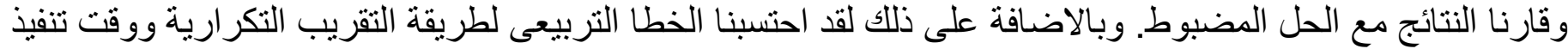
البرنامج.

يوخته

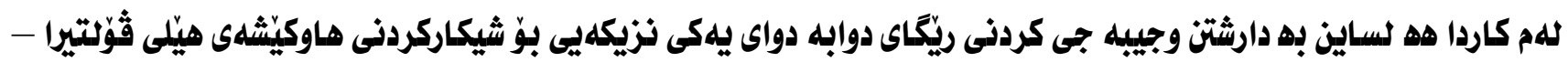

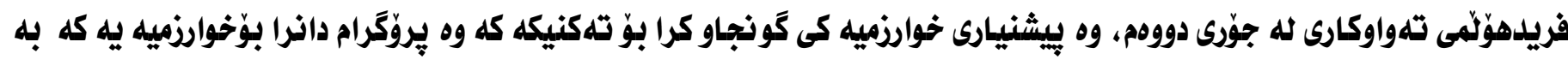

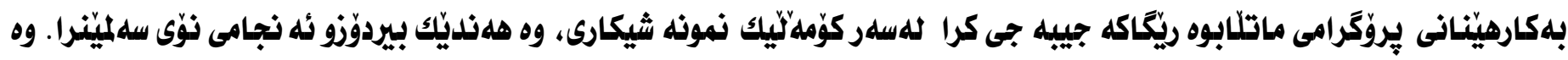

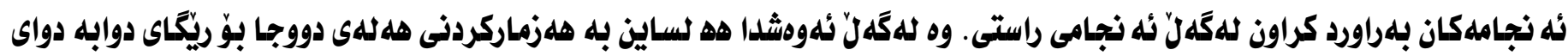

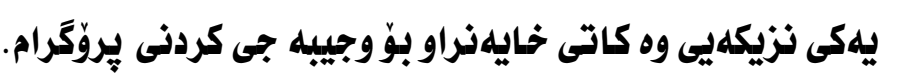

